\title{
Autonomy and Enhancement
}

\author{
G. Owen Schaefer • Guy Kahane • Julian Savulescu
}

Received: 22 April 2013 / Accepted: 1 August 2013 /Published online: 17 August 2013

(C) The Author(s) 2013. This article is published with open access at Springerlink.com

\begin{abstract}
Some have objected to human enhancement on the grounds that it violates the autonomy of the enhanced. These objections, however, overlook the interesting possibility that autonomy itself could be enhanced. How, exactly, to enhance autonomy is a difficult problem due to the numerous and diverse accounts of autonomy in the literature. Existing accounts of autonomy enhancement rely on narrow and controversial conceptions of autonomy. However, we identify one feature of autonomy common to many mainstream accounts: reasoning ability. Autonomy can then be enhanced by improving people's reasoning ability, in particular through cognitive enhancement; given how valuable autonomy is usually taken to be, this gives us extra reason to pursue such cognitive enhancements. Moreover, autonomy-based objections will be especially weak against such enhancements. As we will argue, those who are worried that enhancements will inhibit people's autonomy should actually embrace those enhancements that will improve autonomy.
\end{abstract}

Keywords Autonomy · Enhancement · Reasoning · Cognition

G. O. Schaefer

Lincoln College, University of Oxford,

Turl Street, Oxford OX1 3DR, UK

G. Kahane $\cdot$ J. Savulescu $(\bowtie)$

Uehiro Centre for Practical Ethics, Faculty of Philosophy,

University of Oxford,

Suite 8, Littlegate House 16/17 St Ebbe's St,

Oxford OX1 1PT, UK

e-mail: julian.savulescu@philosophy.ox.ac.uk

\section{Introduction}

Recent years have seen a rapid advancement in research that could, now or in the near future, allow for significant enhancement of various human capacities. Yet some scholars have leveled objections at various means of biomedical enhancement, in particular the alteration of the genes of prospective children. The perceived ills of biomedical enhancement include diminishment of authenticity [1], widespread social stratification and inequity [2], threats to human nature and dignity [3, 4], and hubristic rejection of 'the given' [5]. One further objection, the focus of the present paper, is that biological enhancement can potentially undermine the autonomy of the enhanced individuals. Thus, Jurgen Habermas writes, "Eugenic programming of desirable traits and dispositions...gives rise to moral misgivings as soon as it commits the person concerned to a specific life-project or, in any case, puts specific restrictions on his freedom to choose a life of his own" [6, p. 61]. Deena Davis has similarly objected to some enhancement of children on the grounds that it limits children's future options $[7,8]$.

Yet these autonomy-based concerns overlook the potential versatility of enhancement technologies. Traditionally, the enhancement debate has focused on improving things like health, longevity, intelligence, beauty or skill; however, as we will argue, autonomy is itself something that can be enhanced. In fact, autonomy enhancement is a typical by-product of one of the interventions Habermas and others object to: cognitive enhancement. This is not to equate autonomy with cognition or reasoning capacity; rather, we will argue that cognition and reasoning capacity can significantly contribute to agents' autonomy. By properly attending 
to how such interventions might improve autonomy, we will not only show how certain objections to genetic enhancement can be resisted, but also present an important yet often-overlooked reason to support research and implementation of certain forms of human enhancement. ${ }^{1}$

In the following, we will argue that interventions to enhance autonomy are feasible on a wide variety of conceptions of autonomy. Then, we will discuss how cognitive enhancement will generally also enhance people's autonomy, no matter what (plausible) theory of autonomy one adopts. Finally, we will discuss how this conclusion can help diffuse some autonomy-based objections to enhancement in general, and genetic cognitive enhancement in particular. This reflects a general strategy for defenders of human enhancement: objections that human enhancement interferes with some important goods can often be met by showing that those goods themselves can be promoted via certain forms of enhancement.

The idea that autonomy can be enhanced is not entirely new. One might try to promote autonomy, for example, by removing common psychological biases [10]. What is new is the prospect that autonomy could be enhanced by biomedical means. Two of us have already pointed out in past work that certain psychological capacities are necessary for autonomy, and could be enhanced using genetic selection [11]. The present paper significantly expands on that earlier argument.

Levy and Juth have recently also addressed the possibility of enhancing autonomy $[12,13]$. However, as will be explained below, their discussion is narrower and less likely to be persuasive to many who do not share their particular views on autonomy. They also do

\footnotetext{
${ }^{1}$ There is more than one way to understand the concept of enhancement (see [9] for an opinionated review). In what follows, we will largely focus on biomedical (and other) interventions that increase the capacities that (we will argue) underpin autonomy in normal healthy individuals. On some conceptions of enhancement, it needs to involve increase in value, or be beneficial to the enhanced person. Since it is widely held that autonomy is valuable, the interventions we will discuss would clearly count as enhancements on this conception. Other, more value-neutral conceptions understand enhancement in terms of improvement in some capacity, when that improvement is above some minimal threshold level. The interventions we consider should also count as enhancements on this approach as well. But in any event, the interventions we will consider would clearly be seen as enhancements by the proponents of the autonomy-based arguments against enhancement that we will consider later in this paper.
}

not directly address the implications of such autonomy enhancement to autonomy-based objections to enhancement. This paper will, then, address important aspects of autonomy enhancement that have not yet received attention in the literature. ${ }^{2}$

\section{The Prospect of Enhancing Autonomy}

\section{Diversity in Accounts of Autonomy}

The primary difficulty in developing a case for enhancing autonomy is that there is deep philosophical disagreement as to what autonomy actually is, to say nothing of why it is valuable.

According to Kantianism, for instance, autonomy is an aspect of the will: it does not just cause one to act, but is also itself uncaused; it, moreover, involves attending to substantive normative principles that give rise to reasons for action [15]. While uncaused causation is not essential to a Kantian framework [16], a substantive conception of the will (and hence autonomy) that involves commitment to certain views of moral reasoning is crucial. It gives rise to a normatively non-neutral picture of autonomy on which autonomous judgment amounts to attending to normative reasons, which in turn ground obligation [17]. That is not to say that one only acts autonomously when one acts morally; rather, autonomous action is defined by something like an attempt to respond to the (normative) reasons that there are, in particular, universalized normative principles.

A competing hierarchical conception of autonomy originates in the work of Harry Frankfurt [18]. While Frankfurt technically did not mention autonomy (instead focusing on freedom), his views are widely taken to be applicable to the concept. Frankfurt drew a distinction between two crucially different sorts of desires: first-order desires, which cover most cases of desiring (desiring to eat, to sleep, to be smart, to win the lottery,

\footnotetext{
${ }^{2}$ It has been suggested by John Harris that cognitive enhancements could effectively work as indirect moral enhancements [14]. Improving autonomy, however, is importantly distinct from enhancing morality. While autonomous action is relevant to morality (in particular, when responsibility is being ascribed), there is not much reason to think that making someone more moral will, as a matter of course, make that person more autonomous. And even if such a case could be made, it will not be explored in this paper.
} 
etc.); and second-order (and higher) desires, which are desires to have a certain desire. Autonomy is a particular sort of coherence between these two forms of desires (or related states like judgments, preferences, plans, etc.). ${ }^{3}$

Another loosely-affiliated group of alternative conceptions of autonomy seek to distinguish themselves from hierarchical views, but retain such views' emphasis on agents' internal psychological integration and immunity from manipulative influences. Beauchamp and Childress [22] in attempting to find a broadly acceptable account, characterize autonomy by intentionality, understanding and absence of controlling influences. Similarly, Bernofsky [23] takes autonomy to consist in competence and individuality. According to these views, a general comprehension of one's actions and the facts germane to that action is central to autonomy. ${ }^{4}$

The diversity of accounts of autonomy presents a difficulty for any attempt to defend the prospect of enhancing people's autonomy. One could appeal to one of the above theories of autonomy, or (more ambitiously) craft a new theory, but then one's account would only be acceptable to that (potentially narrow) group who accept a given controversial account. Those who disagree with the account - and, given the level of

\footnotetext{
${ }^{3}$ Of course, this coherence can and has been be cashed out in a number of different ways by proponents of hierarchical views. David Richards, for instance, refers to autonomy as the capacity to "develop, want to act on, and act on higher-order plans of action which take as their self-critical object one's life and the way it is lived" [19, p. 6]. This emphasis on a life-plan bears some relation to Young's [20] holistic conception of autonomy, but unlike Young, Richards (following Frankfurt) emphasizes the need for one to be able to endorse (rather than simply express or be aware of) one's life-plan. Similarly, Gerald Dworkin argues autonomy is "a second-order capacity of persons to reflect critically upon their first-order preferences, desires, wishes, and so forth and the capacity to accept or attempt to change these in light of higher-order preferences and values" [21, p. 20]. This, importantly, requires that those capacities be independent, to a certain degree-free from distorting influences like deception and manipulation.

${ }^{4}$ There are, of course, further accounts of autonomy besides these. John Christman [24], for example, claims that assent to the process by which one came to have one's dispositions and desires is essential to autonomy, while Robert Young [20] instead conceives of autonomy in terms of the unity of one's life-plans. And Jurgen Habermas [6], whose account will be discussed in greater detail below, interprets autonomy as self-authorship, the capacity to guide one's own emotional and intellectual growth. There is also a dispute among various authors as to whether autonomy is a feature of persons [20, 21], actions [22, 24], or the will $[15,17]$.
}

disagreement in the literature on autonomy, there will be many - would not be convinced.

This presents a serious problem for the few previous attempts to develop the idea that autonomy could be enhanced. This idea is discussed by Niklas Juth [12] and Neil Levy [13], but their understanding of autonomy conflicts with many theories of autonomy. In particular, Juth and Levy both endorse efficacy (ability to act on and carry out one's goals and desires) as a necessary condition of autonomy, but this condition (which has a notably external character) is not found in any of the mainstream conceptions of autonomy surveyed above. Autonomy is, on those views, clearly distinct from instrumental rationality. In addition, Juth's account faces difficulties because another of his specified conditions for autonomy, authenticity, is a notoriously vague and underspecified notion, making the task of elucidating how autonomy might be enhanced quite difficult. Levy's account is more succinct, but his discussion of autonomy enhancement is somewhat undeveloped (it appears in the context of a broader discussion of removing cognitive biases) and does not fully engage with the wider literature on autonomy. These difficulties not only make Juth and Levy's accounts somewhat less persuasive, but also make it difficult to use them when engaging with critics of enhancement; if one is not clear about what autonomy is, it will be difficult to adjudicate whether enhancement infringes on autonomy.

Two of us [25] have gestured towards the psychological alterations that would be necessary for enhancement of autonomy, but our discussion is schematic and brief. What we need, then, is not a full account of autonomy, but rather a substantive understanding of the features common to all (or at least most) plausible conceptions of autonomy. Once these features have been identified, one generally-acceptable way to enhance autonomy would simply be to improve on those features. The result will be an account of autonomy enhancement that should be convincing no matter what particular theory of autonomy one adopts. This method of finding an overlapping consensus is quite common in applied ethics, and can have very useful results; for example, there is a great deal of philosophical disagreement over the nature of wellbeing, but discussions of practical improvements in people's well-being is possible because of the overlapping consensus among those various theories when it comes to many substantive questions about 
well-being. As we will see, a similar sort of substantive overlapping consensus can be found for autonomy and applied to the particular issue of enhancement. ${ }^{5}$

\section{Overlapping Consensus: The Theoretical Approach}

Despite the various theoretical differences discussed above, there is considerable agreement among the various theories of autonomy explicated above concerning certain features of autonomy. These features will not constitute a full account of autonomy. Rather, the features are generally-accepted constituents of autonomy, such that improving on those features is generally taken to improve people's autonomy. Enhancement of autonomy will be shown to be plausible, then, insofar as those features can be enhanced.

What do the leading conceptions of autonomy have in common? At the broadest level, autonomy is related to notions of freedom and self-determination. However, these notions are too vague to be useful in the present context. More usefully, various views endorse, in different ways, the idea that autonomy is crucially related to people's reasoning, deliberative and evaluative capacities. This relation between autonomy and cognition is generally positive - greater reasoning, deliberation and evaluation typically leads to greater autonomy. There are a number of ways one could cash out the relationship between reasoning and autonomy on different accounts. For simplicity of analysis, we will focus on three: deductive/logical competence, comprehension (including the avoidance of false beliefs), and critical analysis. The object of these capacities can be both external (arguments and claims made by others or evidence observed) and internal (one's own reasons, arguments, values, etc.).

We can see this common strain by attending to several leading theories of autonomy. Consider first hierarchical theories like Frankfurt's, which are (roughly) concerned with the coherence of higherorder and lower-order desires. Strictly speaking, desires are non-cognitive. However, reasoning capacities can be crucial in resolving potential conflicts. In the first place, some logical abilities will be needed to

\footnotetext{
$\overline{5}$ This isn't to deny that a strong case cannot also be made that autonomy can be enhanced by other means, in line with some particular account of autonomy (say, by enhancing moral sensibility or self-control). But these ways of enhancing autonomy will fall outside the overlapping consensus, and therefore likely to be more controversial.
}

recognize that there is a conflict in need of resolution. An unwilling addict (a paradigmatic instance of failure of hierarchical autonomy) must recognize the conflict between the lower-order desire to consume some substance and the higher-order desire to cease consumption of that substance (and comprehend the greater importance of the higher-order desire) in order to begin to overcome her condition. ${ }^{6}$ Further, attempting to discern appropriate strategies for bringing one's various desires into line will require critical analysis of a range of options. And more nuanced hierarchical theories (e.g., [21]) note that autonomy requires certain reflective, self-critical capacities that lie well within the scope of potential cognitive enhancements.

Autonomy is even more naturally amenable to cognitive enhancement on theories that focus on competence and individuality. The idea of competence, though vaguely defined, is clearly linked to people's ability to reason - their ability to properly comprehend the options ahead of them, evaluate different options, deduce appropriate courses of action, weigh consequences, etc. And independence of thought is not just a matter of ignoring the opinions of others; it requires individuals to have sufficiently developed capacities to assess a wide range of topics and situations. The more one can engage directly with some problem, rather than rely on the perspective of others, the more one can claim one's ideas were generated autonomously.

It is also clear how cognitive improvements are a boon to unified life-plan theories like Young's. Forming a coherent picture of one's life requires a large complement of reasoning abilities such as adequate internal reflection, accurate comprehension of the relevant facts of one's life, the ability to critically evaluate different plans and deduce a course of action from relevant premises. The clearer and more discerning picture one has of one's life-plan, the better able one will be to enact that plan. Furthermore, forming a broad-based life plan is a complex organizational task. Reasoning can aid in planning, consideration of consequences and critical reflection of the various facets of one's chosen approach. Such improvements can help ensure that one's chosen course of life is truly one's own.

On none of these views is reasoning and deliberative capacity sufficient for or identical with autonomy. And each view involves a slightly different way in which

\footnotetext{
${ }^{6}$ This is just a restatement of the familiar idea that the first step to overcoming an addiction is recognizing one has a problem.
} 
reasoning abilities affect autonomy. Nevertheless, it is reasonable to interpret each as implying that reasoning and deliberation can contribute to autonomy. Conversely, each view implies that inhibiting someone's ability to reason and deliberate would inhibit autonomy.

For those who hold the related view that reasoning ability affects autonomy only insofar as there is a threshold of reasoning ability necessary for someone to be an autonomous agent, a modified approach can be taken. Being brought above the threshold for autonomy by improving one's reasoning abilities would count as an enhancement of autonomy, while being brought below the threshold by inhibiting someone's reasoning ability would be an inhibition of autonomy. Where precisely that threshold is will not be discussed here; but as long as there is such a threshold, enhancing autonomy by improving some beings' reasoning ability will at least in principle be possible. ${ }^{7}$

\section{Overlapping Consensus: The Case-Based Approach}

If the preceding theory-based defense of the strong relationship between reasoning capacity and autonomy is unpersuasive, consider instead a case-based approach. Much of the debate over autonomy is centered on making sense of a few paradigmatic cases of autonomy inhibition or violation, in order to be able to determine whether autonomy was violated or inhibited in non-paradigmatic cases. Here, we will highlight four such cases: brainwashing, psychological manipulation, deception, and lack of self-awareness. (See, e.g., [19-24, 26]) What is interesting about these cases is that they operate internally. That is to say, they work by affecting individuals' internal psychology and ability

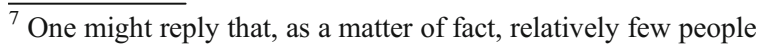
are below the relevant threshold, so autonomy enhancement is not relevant to the vast population. But such a view would implausibly imply that very few acts of deception, manipulation, restriction, and so on actually reduce autonomy. More generally, this suggestion overlooks the extent to which a large variety of factors such as internal biases and external distortions work to reduce people's ability to govern their own lives. For such a view to be plausible, such influences should at least count as violations of autonomy, even if they are not seen as literally reducing autonomy. But this merely reframes the enhancement of autonomy as the prevention of autonomy violations (deception, manipulation, etc.), leaving our main argument intact. While we will not focus on these alternative ways of conceiving of autonomy enhancement in this paper, our arguments should apply mutatis mutandis to such proposals. We are grateful to a referee for pressing us on these points.
}

to reason properly. Brainwashing and psychological manipulation aim at altering (fundamentally, in the case of brainwashing) people's beliefs and desires through non-rational and (usually) non-consensual means. Deception aims at creating false beliefs, thereby inhibiting individuals' ability to reason soundly. A lack of self-awareness prevents people from attending properly to their own beliefs, desires, interests, and so on. Moreover, all these internal effects relate directly to people's ability to reason and deliberate properly. So, even if one rejects the idea that most plausible concepts of autonomy include the idea of reasoning capacity, in practice, many paradigmatic autonomy violations crucially involve inhibition of reasoning and deliberation. There might be other ways to violate someone's autonomy not related to reasoning and deliberation, but all that is necessary for the present argument is to show that many paradigmatic cases of autonomy inhibition work by inhibiting reasoning and deliberation. Thus, preventing such inhibitions by improving people's reasoning and deliberation should have the effect of enhancing autonomy, insofar as there would be fewer instances when one's autonomy will be inhibited. ${ }^{8}$

Recognition of this common feature can help identify precise means by which autonomy could be plausibly enhanced, without being committed to a particular (and likely controversial) conception of autonomy. That is not to endorse a particular conception of autonomy as a certain capacity for reason; rather, such a capacity is a relatively uncontroversial feature of autonomy. Most will accept that reasoning capacity is necessary for autonomy, and moreover that inhibition of such a capacity will generally inhibit autonomy. Thus this overlapping consensus view of autonomy suggests a broadly acceptable means of autonomy enhancement: improving people's reasoning abilities, especially their abilities to resist deception and

\footnotetext{
${ }^{8}$ It goes without saying that improvement in cognitive capacity will often have different significance on different conceptions of autonomy. But this isn't a problem. In a similar way, something - say deep personal relationships-will promote well-being in very different ways on objective, desiresatisfaction and hedonic conceptions of well-being. But if this falls within the overlapping consensus, then these opposing conceptions can all still agree that promoting deep relationships is a good thing. Notice also that our claim about overlapping consensus doesn't assume that different accounts of autonomy target a single unified phenomenon. We only claiming that they can all agree that certain core features play an important role in autonomy.
} 
manipulation. ${ }^{9}$ And, conversely, it implies a straightforward way that autonomy can be inhibited: impairing someone's general reasoning capacities.

It could be objected that we have overlooked how improving reasoning could actually reduce autonomy. One such case would involve decisional paralysis. Perhaps by improving someone's reflective and deliberative capabilities, they will engage overmuch in reasoning rather than making a decision. Insofar as this inhibits decision-making, autonomy could plausibly be impaired. This is a legitimate worry, and one that our account can accommodate by accepting that there may be an appropriate 'mean' of certain reasoning capacities. Above a certain level, some enhanced capacities may indeed actually inhibit autonomy. Still, there is some reason to think that, for most reasoning capacities, people are generally below the ideal mean. There is not adequate space here to fully motivate that claim, but running the reversal test [28] can help: would we think it generally an improvement in autonomy to reduce certain reasoning capacities? It seems unlikely for the general population, but perhaps there are some individuals who do indeed overthink things and thus could have improved autonomy by reducing certain deliberative inclinations. Moreover, it is important to recognize that over-analyzing can itself be subject to cognitive regulation. An integral part of proper reasoning (comprehension, in particular) is the capacity to calculate the opportunity costs of further deliberation on one issue and the ability to recognize when it is appropriate to deliberate further, and for how long. If that is the case, the proper solution is not be to simply inhibit deliberation, but rather improve people's ability to calculate when deliberation is important to achieving their goals or values, and at what point it should be foregone. Insofar as that helps avoids paralysis, it

\footnotetext{
${ }^{9}$ One could argue that there is strong overlapping consensus on certain aspects of autonomy that do not relate directly to internal mental functioning. For instance, coercion is frequently taken as a paradigmatic case of autonomy inhibition, but it generally has little to do with interfering with or altering someone's mental processes. But this view is not universally accepted; Benjamin Sachs [27], for instance, has proposed that coercion is best cashed out in terms of threats, not in terms of autonomy inhibition or violation. In any case, even if there are additional, widelyagreed upon aspects of autonomy (such as immunity from coercion), this does not militate against our case that enhancement of autonomy via improvements to reasoning ability is quite possible.
}

would be another way to improve autonomy by improving certain reasoning capacities. ${ }^{10}$

While we have attempted to remain neutral, for the most part, between a number of popular competing conceptions of autonomy, our account is to be sure not completely neutral. In particular, the preceding discussion did involve some evaluation of how different processes affect reasoning ability and, in turn, autonomy. These evaluations are not in themselves normative, but if one takes the (widely-accepted) view that autonomy is valuable, it does indeed have normative implications, some of which are discussed below. This is a welcome outcome - we take a roughly neutral stance towards different notions of autonomy in order to put forward a generally-acceptable account, rather than avoid particular normative judgments or implications. ${ }^{11}$

\section{Cognitive Enhancement as Autonomy Enhancement}

It is important to note that cognitive enhancement is not necessarily the only way to enhance autonomy; other interventions, such as the direct reduction of conformist impulses or improving people's imagination or creativity, could, on various accounts, also improve autonomy, and thereby make for an even stronger (but also more controversial) case for autonomy enhancement. We focus on cognitive enhancement because it is amenable to the overlapping consensus we have developed, it is feasible, and it ties in directly to some of the examples cited by those who worry about autonomy violations in enhancement.

With this in mind, we are now in a position to explain how cognitive enhancements can generally enhance autonomy. Recall two related features of the overlapping consensus on autonomy above: particular interventions such as brainwashing, deception and manipulation inhibit autonomy, and broader reasoning ability (including logical competence, comprehension and critical analysis) is a crucial component of autonomy. Cognitive enhancement shows significant promise both in improving the latter

\footnotetext{
${ }^{10}$ Notice, finally, that an increase in cognitive capacity needn't imply an increase in how often and how long one deliberates. What it implies is that when one deliberates, that deliberation would be improved in certain respects.

${ }^{11}$ A related point applies to the suggestion, made to us by an anonymous referee, that substantive normative assumptions might be written into the very notion of improvement in cognitive capacities such as the capacity for reasoning-perhaps 'good reasoning' cannot be understood in purely procedural terms. Even if this is correct, this needn't be a problem so long as the relevant normative assumptions are widely shared.
} 
features and in helping resist such autonomy inhibiting interventions; and insofar as it improves on those features, it will also enhance autonomy. Now different autonomy theorists might argue for different particular means by which cognitive enhancement can lead to improvement of autonomy; for instance, life-plan theorists may think that memory enhancements will be relevant to autonomy while hierarchical theorists ignore it. Still, our approach brings various views into relatively close alignment, to the point that even if disagreements in theory persist, practical consensus over the use of some forms of cognitive enhancement to improve autonomy appears possible.

Furthermore, it may be that only certain aspects of cognition will be relevant to the sort of reasoning central to autonomy. Improving people's ability to perform certain cognitive tasks such as carrying out complex mathematical equations or recalling the meanings of obscure, infrequently-used terms (popular aspects of many standardized tests) would have little to do with autonomy on a number of accounts. However, other aspects of cognition are more relevant. We have already discussed how logical competence, a key aspect of cognition, plays an important role in various accounts of autonomy. In addition, pattern recognition assists in properly comprehending the way the external world, as well as one's own mind, functions. Linguistic ability is needed to understand and communicate with others, improving people's ability to critically analyze different opinions and views. Even improving memory (a central focus of recent research on cognitive enhancement; see, e.g., [29]) can ensure people have correct comprehension of the world and themselves, an important component of autonomy [30] that will again improve deliberation and self-reflection. ${ }^{12}$ Improving

\footnotetext{
$\overline{12}$ As discussed in relation to reasoning above, increasing certain cognitive functions beyond a certain optimal point may ultimately compromise autonomy. For such an example in the context of memory, see Luria [31]. But it seems to us doubtful that most individuals are beyond that point and so improving cognitive functions will, for most people, be an improvement in autonomy. Relatedly, cognitive enhancement may sometimes reduce autonomy by pushing people even further in some mistaken direction - for example, if many of a person's basic beliefs are false, cognitive enhancement may just lead her to develop a more elaborate false system of beliefs, which will in turn reduce her autonomy. This, however, seems to us a weak argument against improving cognitive capacity to enhance autonomyotherwise this point would also cast doubt on the entire enterprise of education. On the contrary, improved reasoning can be generally expected to make beliefs more accurate, even if it sometimes leads one astray. Our claim is, after all, not that cognitive improvements will invariably enhance autonomy, just that they generally will. We are grateful to an anonymous referee for pressing this point.
}

comprehension, and thus the accuracy of beliefs, is particularly important for autonomous choice. Autonomy involves choice between options for what one judges one ought to do. To appreciate the nature of options, we need true beliefs about their nature. If you have inaccurate beliefs about, for instance, living in England (say, you think the climate is very pleasant and you like sunny weather), you are not really choosing to live in England because it is best for you.

There is also good reason to think that cognitive enhancement can help mitigate some of the influences that reduce autonomy. Psychological manipulation can take a variety of forms, but at least one potential mechanism is through conformity: people adopt potentially fallacious or misguided beliefs or desires not on their own merits but in virtue of the beliefs and desires of the surrounding community. ${ }^{13}$ One of the classic demonstrations of this phenomenon comes from Asch [33] who famously demonstrated that people can be made to give obviously false answers to questions on subjects such as the relative length of two lines when their peers give such obviously false answers. A number of follow-up studies have shown that greater intelligence is strongly correlated with resistance to conformity in such circumstances [34-36]. Such resistance to conformity is not merely a matter of expertise; those studies found that intelligence is also correlated with lower conformity with the unconventional or counterintuitive normative opinions of one's peers. Nancy Rhodes and Wendy Wood have postulated that higher intelligence impedes influence insofar as more intelligent people have greater working knowledge and "possess the ability to critically evaluate and reject all but the most cogent messages" [37, p. 158]. If that is the case, then cognitive enhancement would likely help prevent at least some forms of manipulation (though of course even the cognitively enhanced would likely still be susceptible to manipulation to some degree) and preserve independence of thought. ${ }^{14}$

\footnotetext{
${ }^{13}$ This is akin to what Mill had in mind when he emphasized the importance of "originality" [32, p. 121]. Mill's distinction between higher and lower pleasures is based on the comparison of those who have experienced both. Mill also thought that vivid imagination of alternatives was sufficient to make such a comparison (On Liberty, [32], p.10). This psychological capacity of imagination also varies between individuals and could in principle be enhanced.

${ }^{14}$ One relevant and intriguing consideration is the considerable evidence that views and attitudes that appear to have a strong genetic basis are also the most resistant to social pressures. See Tesser [38].
} 
Unfortunately, as far as we are aware, similar studies have not been carried out to test whether improved cognitive capacities reduce vulnerability to deception. Still, it stands to reason that cognitive enhancement would in fact have a similar effect on susceptibility to deception. In the very least, cognitive enhancement will promote the accuracy of people's beliefs, which could subvert attempts by others to instill contrary false beliefs. Moreover, improving reasoning capacities could help improve people's ability to effectively evaluate the claims of others and recognize fallacious arguments. So, insofar as deception and manipulation are inimical to autonomy (a claim accepted by all the mainstream views on autonomy) cognitive enhancements could be very effective means of improving autonomy.

Some might find this conclusion unsettling. It implies that more intelligent people are more autonomous; one might think that, therefore, we would be more justified in making decisions on behalf of less intelligent people, as their autonomy is diminished. This worry, however, is mistaken. In the first place, most theories of autonomy already admit of degrees - some adults are more autonomous than others, and each person more autonomous at some times, in some circumstances. If this conclusion is problematic for our view, it is also a problem for most mainstream views of autonomy. Our view should not, then, be any more disturbing than those views. Also, while intelligence might improve autonomy, it is clearly neither sufficient for nor the only factor that improves autonomy. Moreover, differing levels of autonomy do not justify differing levels of paternalism; the solution to diminished autonomy is generally not to make decisions on behalf of individuals, but instead to help them be more autonomous in their decision-making - in other words, to enhance their autonomy. This is one aim of education, which is itself a cognitive enhancer, and the commonest means by which autonomy is enhanced. ${ }^{15}$

\footnotetext{
${ }^{15}$ One interesting implication of this view is the converse - just as certain interventions that affect cognitive ability can enhance autonomy, other interventions could inhibit it. For instance, one might think it is important to make society more cooperative; one means of doing so would be to increase people's conformity and susceptibility to social influence. While there may be beneficial outcomes from such an intervention (such as greater provision of public goods or less social strife), it is important to note it would have significant costs as well, in terms of a diminishing of people's autonomy. Another example of autonomy-inhibiters would be pollutants that cause cognitive defects, such as lead exposure through water, paint and other sources [39]; in addition to the health ramifications, one could raise the further objection that such pollutants lead to long-term reduction of people's autonomy.
}

As we have seen, cognitive enhancement generally has the effect of enhancing autonomy. ${ }^{16}$ This finding is interesting in itself and, when combined with the widely-accepted value of autonomy, it also gives us additional reason to pursue and promote biomedical cognitive enhancement. And, as the next section will show, it has the added advantage of being resistant to several autonomy-based objections to enhancement. We of course do not deny that environmental influences can make an important difference to cognitive capacity (see, e.g., [40]), and thus also autonomy, if our argument is correct. It is a further empirical question whether biomedical means are feasible and efficacious means to this end. But it is most likely that the best result would involve a combination of environmental and biomedical strategies.

\section{Overcoming Autonomy-Based Objections to Genetic Enhancement}

As mentioned in the Introduction, a number of objections to biomedical enhancement have been raised in the literature. Most of those objections would apply with equal force against autonomy enhancement as against interventions that did not enhance autonomy. However, autonomy enhancement does bear directly on one central objection: enhancement via embryonic genetic manipulation ${ }^{17}$ violates the autonomy of the future child.

Whether or not such autonomy-based objections are sound for other forms of enhancement, using genetic manipulation to enhance autonomy along the lines discussed above can avoid, or at least blunt, their force. Recent studies have made significant progress in identifying genes correlated with greater intelligence. (see, e.g., [41-44]) Based on such findings parents may, in

\footnotetext{
$\overline{16}$ Those who wish to emphasize that cognitive capacity isn't on its own sufficient for autonomy may prefer to instead speak of enhancing opportunity for autonomy rather than autonomy itself. But for our purposes here this distinction is unimportant.

${ }^{17}$ These same critics also object to embryo selection for the purpose of enhancement, but this view faces the non-identity problem. If a couple selects one embryo over another, then strictly speaking no individual's autonomy could be violated. For if they had not undergone the selection, then the selected-for embryo would likely not have existed; and it is difficult to argue that one would respect the autonomy of a future child by not bringing him or her into existence. There is not space here to address this issue thoroughly, and so we will set it aside.
} 
the near future, be able to use genetic manipulation to improve people's cognitive capacities - which, given the above arguments, would lead to enhancement of autonomy. Of course, genetic manipulation is not the only way to enhance autonomy; other biomedical interventions such as pharmaceuticals, or more traditional means such as education, could have the effect. However, genetic manipulation is worth focusing on because it is uniquely controversial and at the center of several authors' concerns about enhancement.

We will now discuss how autonomy enhancement can help address two objections to enhancement via genetic manipulation, one concerning a child's right to an open future, and the other concerning subversion of the process of autonomous self-directed formation of traits.

\section{The Open Future Objection}

The first objection comes from Dena Davis, who adapts Joel Feinberg's conception of a child's right to an open future as an objection to certain kinds of genetic enhancement. ${ }^{18}$ The right to an open future, says Feinberg, is not an existent right, but rather a right-in-trust; "The violating conduct guarantees now that when the child is an autonomous adult, certain key options will already be closed to him." [45, p. 77] One paradigmatic example of violating a child's right to an open future would be preventing the child from attending school, thus shutting off (or at least seriously inhibiting) various career options that require a certain basic level of education. Feinberg considers this to be a sort of autonomy-right; while some autonomy theorists might disagree about this classification, the right is at least rather plausible. Indeed, preventing a child from going to school also inhibits autonomy on our overlapping consensus account as well, insofar as it deprives the child of the intellectual resources that contribute towards personal autonomy.

Davis [7, 8] expands Feinberg's argument to cover not only existent children, but potential children as well-including embryos potentially the subject of genetic manipulation. This argument does not assume that embryos have any moral status; however, there is a

\footnotetext{
${ }^{18}$ Davis focuses on genetic selection, but her arguments also apply to genetic manipulation; we will address the latter for reasons explained in the previous footnote, but our arguments should apply mutatis mutandis to her concerns about genetic selection.
}

sense in which any future child resulting from genetic manipulation has a right against parents taking actions at any point-indeed, even before the child was conceived - that would inhibit the child's options later in life. Thus, according to Davis, using genetic manipulation to select for a disability like deafness violates the child's right to an open future insofar as such manipulation inhibits the option to easily integrate into the hearing community [7]. ${ }^{19}$

This argument is vulnerable to a number of objections. Many non-objectionable parenting decisions alter a child's options in the same way, and choosing between various mutually-exclusive options (where to send a child to school, what clothes to buy, what food to prepare, etc.) is inevitable [46]. It is not clear why similar options-alteration via enhancement would be especially problematic.

The argument becomes especially implausible in the case of autonomy enhancement. The main plausible options that are shut off by having more autonomy (such as integration into a very manipulative community or one that disvalues reasonable thought) seem patently objectionable, even harmful, and could not provide a serious reason against enhancing autonomy. That is to say, the options that are shut off are not particularly valuable - manipulative communities are generally not worth joining. In addition, being autonomous is integral to taking full advantage of various options; enhancing autonomy should directly promote the number and value of a child's options, not restrict them. Even if increased autonomy remove a handful of options (such as joining a community that disvalues autonomy) from the menu, many more will be opened up (more career opportunities, better management of resources to obtain what one wants, and even greater ability to discern how to integrate into a wide variety of communities, etc.)

A more indirect way that genetic manipulation can violate a child's right to an open future is through the creation of parental expectations and entitlements. Because genetic manipulation is, at present, somewhat costly, parents who engage in such manipulation in order to direct their children's lives in a certain way could reinforce and bolster parents' unwillingness to

\footnotetext{
${ }^{19}$ As an anonymous reviewer has pointed out to us, one problem with this example is that it might also be argued that being hearing also limits one's options by making it harder to integrate into the deaf community.
} 
accept the child deviating from the selected life-plan [8], see also [5]. Suppose, for instance, that parents perform genetic manipulation in order to enhance their child's athletic ability, because they very much want their child to be an elite athlete. While this enhancement could be seen as opening up certain options (improving the child's ability to actually become an elite athlete), the parents may also feel so invested in the child's athletic career that they would not tolerate or accept the child choosing a different career path. The enhancement, then, indirectly creates an environment in which a child's options are limited — what Sandel [5] calls "hyper-parenting".

Davis has similar worries about cognitive enhancement: "Even traits that are useful for all life plans (such as intelligence) may be chosen with very particular life plans in mind, with expectations that may restrict the child's future freedom" [8, p. 26]. So, for example, parents might choose to genetically enhance a future child's autonomy by making the child more immune to manipulation and deception in order to prevent the child from belonging to a political party they believe to be particularly manipulative and deceptive. We might worry that such investment creates an environment in which the parents would become even more intolerant of any inclination the child might later have to join such a political party, inhibiting the child's ability to join that political party.

Still, it is difficult to imagine such parents having a specific life-plan in mind for their children in the first place. The political party case mentioned above seems rather unrealistic; the most plausible reason parents would attempt to enhance their child's autonomy is to expand life-options, which can hardly be objectionable. And even if the parents did have a specific lifeplan in mind, their commitment to the importance of the capacities that underpin autonomy (evidenced by the decision to enhance them) indicates a similar commitment to tolerance of various options, thus minimizing the risk of restricting their child's right to an open future.

Even for parents who end up enhancing their child's autonomy as an unintended side-effect of cognitive enhancement for other purposes, the case for an indirect inhibition of the child's right to an open future is rather weak. Such enhancements will generally have the direct effect of increasing, rather than decreasing, a child's options. In general, inhibitions and violations of autonomy restrict options; they lead an individual to unreflectively adopt a certain path, perhaps one determined by others, reducing a person's ability to take seriously alternatives that might, indeed, be better. By reducing such restrictions, enhancement of autonomy should open up a whole host of options that were not as easily accessible before. In fact, it should help prevent the sort of parental manipulation Davis is concerned about; a child whose autonomy is enhanced will better be able to resist the effects of the intolerance of his or her parents' expectations, and more likely to pursue his or her own interests in spite of the parents' planseven if the parents' aim in seeking this biomedical intervention had nothing to do with autonomy. ${ }^{20}$ Moreover, the risks of parental expectations constraining the child's future options have to be weighed against the direct benefits of the enhancement on autonomy; in light of the wide consensus over the value of autonomy discussed above, it seems that autonomy will likely weigh very heavily indeed.

\section{The Communicative Objection}

The second objection comes from Jurgen Habermas, in The Future of Human Nature [6]. This objection is intimately related to Habermas' more general moral and political focus on the importance of communication and discourse. It also finds its roots in Kantian moral theory, premised on the idea that people must be treated as an end in themselves, never merely as a means. Habermas extends this thought to the process by which an individual is formed: it is also important, he argues, that people not be instrumentalized (treated merely as a means) via genetic manipulation. This instrumentalization is contrasted with a communicative process, which involves the ability to assent or dissent to (and critically engage with) whatever intervention is being proposed. The upshot is that inculcation of values and expectations is permissible, so long as adequate space is given for the child to reflect on such inculcation and guide the direction of his or her own intellectual growth. Autonomy, then, is crucial to people's very identity and sense of self.

\footnotetext{
${ }^{20}$ This claim should not be taken too far. An extremely controlling or manipulative parent will likely be able to find ways to inhibit a child's autonomy no matter how much cognitive enhancement has been employed. The point here is just that cognitive enhancement can provide some resistance to these attempts.
} 
Genetic manipulation is seen as problematic because it occurs prior to the child being able to engage with and provide input on the intervention. According to Habermas,

"Eugenic interventions aiming at enhancement reduce ethical freedom insofar as they tie down the person concerned to rejected, but irreversible intentions of their parties, barring him from the spontaneous self-perception of being the undivided author of his own life" [6, p. 63].

Genetic manipulation violates autonomy not only because it restricts certain options, but also because the child himself or herself had no input or communicative engagement with that restriction. This in turn inhibits the autonomous formation of the child's identity, which Habermas takes to be of great importance. ${ }^{21}$ Habermas does, however, make an important concession: he accepts that genetic manipulation in order to prevent grave illnesses in future children may be permissible because it is reasonable to presume that the resultant child would agree to the procedure. ${ }^{22}$

It is true that genetic manipulation is undertaken without the communicative input of the child, thus precluding the possibility of discursive engagement that Habermas takes to be crucial. And such communicative engagement with even young children is indeed important. Failing to engage in such communication with one's children might inhibit autonomy on our account as well, insofar as such would fail to properly cultivate the child's reflective and deliberative capacities; and similarly, any genetic manipulation aimed at preventing that communicative process later on would be difficult to justify.

Yet, genetic manipulation seems far less problematic when the manipulation is undertaken in order to enhance autonomy. In Habermas's own view, autonomy is central to human agency. Moreover, enhancement of autonomy via improved cognitive abilities

\footnotetext{
${ }^{21}$ Sandel [5] has somewhat similar concerns about the excessive control genetic manipulation affords parents over their children; however, he identifies the problem as less about inhibiting children's autonomous formation and more about parental rejection of children's given natures.

${ }^{22}$ Habermas limits his objections to third-party prenatal enhancements. However, Peter Herissone-Kelly [47] has argued that the thrust of Habermas's argument (enhancement interferes with self-discovery and self-formation) applies to adult selfenhancement as well, insofar as self-enhancements undermine the reflexive agency that makes many activities valuable.
}

would improve the child's ability to communicatively engage with the world. So, for instance, by making someone more self-aware and cognizant of the various factors that influence them, they will be better able to guide and direct their own growth. ${ }^{23}$ And even if Habermas were still reluctant to endorse the positive promotion of autonomy, it is harder to see how he would resist interventions aimed at reducing violations of autonomy. Indeed, enhancing autonomy would be a powerful weapon against instrumentalization, insofar as it could prevent people from having a distorted view of the world and themselves. As Habermas takes the capacity to personally engage with and provide input into the factors that shape one's life to be so central, he should embrace autonomy enhancements as a useful tool to improve that capacity. ${ }^{24}$

Furthermore, Habermas accepts that some prenatal interventions, such as those that promote health, are acceptable because of hypothetical consent: it is reasonable to assume that the child would endorse health improvements, as such would not conflict with any reasonable life-plan. Hypothetical consent would arguably also apply to autonomy enhancements - it seems to us similarly implausible to expect that a child would want a less-autonomous life. Otherwise, Habermas's general autonomy-based arguments against other prenatal enhancements would lose its force. After all, if the resultant person would be perfectly fine with having less autonomy throughout their lives, that person would in effect be rejecting the fundamental value that Habermas attributes to autonomous self-discovery. That person would then be rejecting Habermas's view that takes such autonomous self-discovery as essential, making Habermas's other arguments against enhancement that rely on that view inapplicable to

\footnotetext{
${ }^{23}$ This improvement in self-awareness is a natural outgrowth of improved reasoning abilities. Having an understanding of where one's ideas and beliefs come from requires assessment of causal influences and psychological function. While enhancements cannot simply impart that understanding directly, they can assist people's ability to analyze the relevant factors.

${ }^{24}$ Habermas might reply that the benefits to autonomy are beside the point; genetic manipulation is problematic because it is a form of unidirectional socialization, which inherently and problematically alters the child's identity. However, such unidirectional socialization should only be problematic if it ultimately limits the child's ability to engage with and shape his or her identity. Autonomy enhancements have the opposite effect - even if they are unidirectional, they should ultimately enable and promote rather than restrict the self-formation of identity.
} 
this particular individual. ${ }^{25}$ To be sure, a given child might (hypothetically) instead reject the notion that a particular intervention like cognitive enhancement actually improves autonomy - but if our arguments above are correct, this would not in general be a reasonable judgment. It might appear problematic that we impose our own judgments of what is reasonable on future children, but Habermas does precisely the same thing in allowing for medical prenatal treatments. ${ }^{26}$

The Kantian undertones of Habermas's arguments might elicit the following reply: one cannot justify violating someone's autonomy on the basis that the violation would overall promote the autonomy of that individual, just as one cannot justify lying to someone on the basis that the lying would (let us suppose) promote the ability of that individual to see through lies in the future. This reflects the idea that autonomy is, first and foremost, to be respected - even if such respect inhibits the promotion of autonomy.

However, it is not clear that genetic manipulation aimed at enhancing autonomy would count as a violation at all, even on Habermas's account. In the first place, Habermas is not (or at least, should not be) primarily concerned with present autonomy-rights as such, but with the effect of a given intervention on a resultant child's ability to shape his or her own life. This approach makes sense in the present case because embryos are not yet autonomous agents - the embryo will only develop the necessary capacities in the future. The issue is thus not about violating the rights of an autonomous agent at the time of the intervention but about limiting/expanding the autonomy of an agent in the future. ${ }^{27}$ In Habermasean

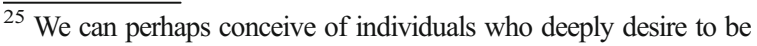
enslaved by others, but it is doubtful that respect for autonomy should make us respect such slavish wishes, or that we should treat a life plan organized around such desires as any more reasonable than a conceivable life plan organized against the desire to be sick and infirm. More reasonably, some might value (say) a life without complex choices even if it is not very autonomous. But even then, increased reasoning/cognition would help people figure out how to 'simplify' their lives most effectively and so would still be worth having.

${ }^{26}$ One might think it is just not sensible to think anyone could object to prenatal health interventions. However, that is actually a distinct possibility-Davis [7], for instance, was motivated in part by the aim of some disabled couples to have children who are similarly disabled. Or to use a more extreme example, an anorexic may regret that her parents did genetically intervene prenatally so that she would be even thinner.

${ }^{27}$ Feinberg's [45] open future account explicated above is an attempt to conceptualize autonomy violations for future individuals. If a defender of Habermas attempts this route, though, our preceding responses to Davis will also apply just as strongly.
}

terms, autonomy is limited when a certain (postnatal) communicative process is inhibited. Even supposing a categorical prohibition on any reduction in the communicative process, autonomy enhancement should be permitted because (as we argue) that communicative process is actually promoted all things considered, rather than inhibited. It is true that the decision to prenatally enhance autonomy is not itself the result of communicative engagement, but that decision will promote many more opportunities for future communicative engagement (along the lines outlined in the first part of this paper). And, in any event, the decision to decline or even prohibit autonomy enhancement would similarly not be the result of a communicative process with the resultant child-no matter what, the parent must make a decision without input from the resultant child, and it is surely better to choose the option that results in more autonomy.

Furthermore, arguing for the strict primacy of an autonomy right against prenatal enhancement over the promotion of autonomy (and, in turn, the communicative process) would undermine the force of the communicative argument, which takes personal control and freedom to be a central value. To hold that even if a given prenatal intervention leads to a vast improvement in a child's ability to direct and shape his or her own life, that intervention should still be prohibited seems to undermines the notion that such self-direction is really of such great importance. $^{28}$

As we have said before, education is one kind of cognitive enhancer that promotes autonomy. This is, to some degree, imposed on young children without communicative dialogue. Habermas does not object to (properly designed) education programs, and rightly so because education is crucially important for the development of autonomy, respecting and promoting the communicative process. But by the same token, Habermas should not object to prenatal interventions that would improve autonomy - including, as we have argued, cognitive enhancements.

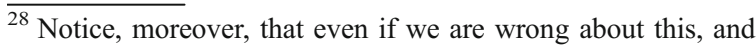
prenatal autonomy enhancement does violate some right, it doesn't follow that it is morally forbidden. It might be a very slight violation of rights, vastly outweighed by the benefit in autonomy - the very value this right is claimed to protect. Few hold the view that telling a toddler a little white lie is forbidden in all contexts.
} 


\section{Conclusions}

While discussion of enhancement has largely focused on more easily-measurable domains such as cognition or health, in this paper we have sought to draw attention to another important potential domain of enhancement: autonomy. It should be clear that, on virtually any mainstream view of autonomy, autonomy can potentially be enhanced. By identifying a general consensus that reasoning ability contributes to autonomy, we further showed how cognitive enhancements (some of which are currently available) can constitute autonomy enhancement as well.

On this basis, we were then able to argue that those who object to enhancement because it inhibits autonomy should nevertheless support, or at least not oppose, the enhancement of autonomy. Habermas, for instance, worries that certain forms of enhancement run afoul of autonomy by barring a person from "the spontaneous self-perception of being the undivided author of his own life" [6, p. 63]. However, we have argued that even on the conceptions of autonomy (such as selfauthorship) that critics of enhancement endorse, it is possible to have enhancements that promote autonomy and thus avoid their critiques.

There has generally been strong support for the enhancement of autonomy in traditional ways. Education, in particular, is a popular way to improve people's ability to reason and thereby govern their own lives. Support for education naturally follows from a strong belief in the value of autonomy. But if our arguments above are correct, similar considerations should also motivate support for novel forms of cognitive enhancement, and reasoning improvement more generally, as a way of promoting autonomy. ${ }^{29}$ Thus, critics of enhancement who take autonomy to be the main reason to resist enhancement programs have reason to not only permit but even support and promote various forms of autonomy enhancement, including enhancement of reasoning capacities. There is not space here to investigate what forms this promotion might take, but it could include promotion of and funding for research on autonomy enhancement, subsidies (as with public education) for autonomy enhancements, and publically

\footnotetext{
${ }^{29}$ Critics may still have other, non-autonomy-based objections to enhancement that are powerful enough to outweigh the value of autonomy enhancement. Still, to the extent that critics find such autonomy-based objections forceful, they should find similarly forceful reasons to support autonomy enhancement.
}

encouraging people to voluntarily undergo such enhancements themselves, as a way to live more autonomous lives.

This paper has reflected a general strategy available to proponents of human enhancement: if a sweeping objection to biomedical enhancement is based on the worry that enhancement will compromise some value, and it can be shown that this value can itself be promoted using biomedical means, then at the very least these forms of enhancement should be prima facie immune to this objection. ${ }^{30}$ Here, we filled in 'autonomy' for 'some value', and so were able to respond to one sort of objection to enhancement. The same strategy could potentially be used to respond to other objections to enhancement. For instance, we could fill in ' $\mathrm{X}$ ' with Sandel's [5] concern, humility in the face of the given. Even if Sandel is right that enhancement in general goes against that humility, he will find it far more difficult to reject enhancements aimed at making people more humble and appreciate life as a gift. We hope that further discussions along this line can help advance the enhancement debate and perhaps overcome some of the seemingly-irreconcilable disputes in the literature.

Acknowledgments We are grateful to several anonymous referees for useful comments. Part of this research was supported by a grant from the Wellcome Trust (WT087208MF).

Open Access This article is distributed under the terms of the Creative Commons Attribution License which permits any use, distribution, and reproduction in any medium, provided the original author(s) and the source are credited.

\section{References}

1. Elliott, C. 2003. Better than Well: American Medicine Meets the American Dream. New York: W.W. Norton \& co.

2. Annas, G.J. 2000. The man on the moon, immortality, and other millenial myths: The prospects and perils of human genetic engineering. Emory Law Journal 49: 753-782.

\footnotetext{
${ }^{30}$ On its own, showing that it is possible to biomedically enhance $\mathrm{X}$ won't yet show that we have positive reasons to do so, just that worries relating to $\mathrm{X}$ would be largely (or completely) defused for this form of enhancement. In the case of autonomy, however, we can make a stronger claim. The value of autonomy enjoys widespread acceptance; it is therefore relatively uncontroversial to draw the normative conclusion that we have positive reason to promote interventions such as cognitive enhancement that will generally improve autonomy.
} 
3. Fukuyama, F. 2002. Our posthuman future. New York: Farrar, Straus and Giroux.

4. The President's Council on Bioethics. 2003. Beyond therapy: Biotechnology and the pursuit of happiness. Washington, DC.

5. Sandel, M.J. 2007. The case against perfection. Cambridge: Belknap.

6. Habermas, J. 2003. The future of human nature. Cambridge: Polity Press.

7. Davis, D. 1997. Genetic dilemmas and the child's right to an open future. The Hastings Center Report 27(2): 7-15.

8. Davis, D. 2009. The parental investment factor and the child's right to an open future. The Hastings Center Report 39(2): 24-27.

9. Savulescu, J., A. Sandberg, and G. Kahane. 2011. Well-being and enhancement. In Enhancing human capacities, ed. J. Savulescu, R. ter Meulen, and G. Kahane, 3-18. Oxford: Wiley-Blackwell.

10. Savulescu, J. 1994. Rational Desires and the Limitation of Life-Sustaining Treatment. Bioethics 8(3): 191-222.

11. Savulescu, J. 2008. Procreative Beneficence: Reasons Not To Have Disabled Children. In J. Thompson and L. Skene (Eds.) The Sorting Society: The Ethics of Genetic Screening and Therapy, Cambridge: Cambridge University Press.

12. Juth, N. 2011. Enhancement, autonomy, and authenticity. In Enhancing human capacities, ed. J. Savulescu, R. ter Meulen, and G. Kahane, 34-48. Oxford: Blackwell Publishing, Ltd.

13. Levy, N. 2012. Ecological engineering: Reshaping our environments to achieve our goals. Philosophy \& Technology (online first).

14. Harris, J. 2011. Moral enhancement and freedom. Bioethics 25(2): 102-111.

15. Darwall, S. 2006. The value of autonomy and autonomy of the will. Ethics 116(2): 263-284.

16. Hill, T. 1989. The Kantian conception of autonomy. In The inner citadel: Essays on individual autonomy, ed. J. Christman, 91-108. New York: Oxford University Press.

17. O'Neill, O. 2002. Autonomy and trust in bioethics. Cambridge: Cambridge University Press.

18. Frankfurt, H. 1971. Freedom of the will and the concept of a person. The Journal of Philosophy 68(1): 5-20.

19. Richards, D. 1981. Rights and autonomy. Ethics 92(1): 3-20.

20. Young, R. 1989. Autonomy and the inner self. In The inner citadel: Essays on individual autonomy, ed. J. Christman, 77-90. New York: Oxford University Press.

21. Dworkin, G. 1988. The theory and practice of autonomy. Cambridge: Cambridge University Press.

22. Beauchamp, T., and J. Childress. 2009. Principles of biomedical ethics, 6th ed. New York: Oxford University Press.

23. Bernofsky, B. 1995. Liberation from self: A theory of personal autonomy. Cambridge: Cambridge University Press.

24. Christman, J. 1991. Autonomy and personal history. Canadian Journal of Philosophy 21(1): 1-24.

25. Savulescu, J., and G. Kahane. 2009. The moral obligation to create children with the best chance of the best life. Bioethics 23(5): 274-290.

26. Lindley, R. 1986. Autonomy. Atlantic Highlands, NJ: Humanities Press.

27. Sachs, B. 2011. Why coercion is wrong when it's wrong. Autralasian Journal of Philosophy 1-20 (online first).
28. Bostrom, N., and T. Ord. 2006. The reversal test: Eliminating stats quo bias in applied ethics. Ethics 116: 656-679.

29. Sandberg, A. 2011. Cognition enhancement: Upgrading the brain. In Enhancing human capacities, ed. J. Savulescu, R. ter Meulen, and G. Kahane, 71-91. Oxford: Wiley-Blackwell.

30. Killmister, S. 2012. Autonomy and false beliefs. Philosophical Studies 1-19 (online first).

31. Luria, A.R. 1987. The mind of a mnemonist: A little book about a vast memory. Cambridge: Harvard University Press.

32. Mill, J.S. 1910. Utilitarianism, liberty \& representative government. London: Dent.

33. Asch, S. 1951. Effects of group pressure upon the modification and distortion of judgment. In Groups, leadership and men, ed. H. Guetzkow. Pitsburgh: Carnegie Press.

34. Cox, F.J. 1960. Some dispositional correlates of conformity behavior. The Journal of Social Psychology 52: 259-268.

35. Crutchfield, R. S. 1955. Conformity and character. The American Psychologist, 191-198.

36. Tuddenham, R.D. 1959. Correlates of yielding to a distorted group norm. Journal of Personality 27: 272-284.

37. Rhodes, N., and W. Wood. 1992. Self-esteem and intelligence affect influenceability: The mediating role of message reception. Psychological Bulletin 111(1): 156-171.

38. Tesser, A. 1993. The importance of heritability in psychological research: The case of attitudes. Psychological Review 100: $129-142$

39. Needleman, H.L., A. Schell, D. Bellinger, A. Leviton, and D.N. Allren. 1990. The long-term effects of exposure to low doses of lead in childhood. The New England Journal of Medicine 322(2): 83-88.

40. Flynn, J.R. 1984. The mean IQ of Americans: Massive gains from 1932 to 1978. Psychological Bulletin 95(1): 29-51.

41. Burdick, K.E., T. Lencz, B. Funke, C.T. Finn, P.R. Szeszko, J.M. Kane, and A.K. Malhotra. 2006. Genetic variation in DTNBP1 influences general cognitive ability. Human Molecular Genetics 15(10): 1563-1568.

42. Deary, I.J., S.E. Harris, H.C. Fox, C. Hayward, A.F. Wright, J.M. Starr, and L.J. Whalley. 2004. KLOTHO genotype and cognitive ability in childhood and old age in the same individuals. Neuroscience Letters 378: 22-27.

43. Harris, S., H. Fox, A. Wright, C. Hayward, J. Starr, L. Whalley, and I. Deary. 2006. The brain-derived neurotrophic factor Val66Met polymorphism is associated with agerelated change in reasoning skills. Molecular Psychiatry 11: 505-513.

44. Houlihan, L.M., S.E. Harris, M. Luciano, A.J. Gow, J.M. Starr, P.M. Visscher, and I.J. Deary. 2009. Replication study of candidate genes for cognitive abilities: The Lothian Birth Cohort 1936. Genes, Brain, and Behavior 8: 238-247.

45. Feinberg, J. 1992. The child's right to an open future. In Freedom and fulfillment: Philosophical essays, ed. J. Feinberg, 76-97. Princeton: Princeton University Press.

46. Robertson, S., and J. Savulescu. 2001. Is there a case in favour of predictive genetic testing in young children? Bioethics 15(1): 26-49.

47. Herissone-Kelly, P. 2012. Habermas, human agency, and human genetic enhancement: The grown, the made, and responsibility for action. Cambridge Quarterly for Healthcare Ethics 21(2): 200-210. 